PROCEEDINGS OF THE

AMERICAN MATHEMATICAL SOCIETY

Volume 126, Number 12, December 1998, Pages 3647-3656

S 0002-9939(98)03353-X

\title{
A RECURRENCE/TRANSIENCE RESULT FOR CIRCLE PACKINGS
}

\author{
GARETH McCAUGHAN
}

(Communicated by Albert Baernstein II)

\begin{abstract}
It is known that any infinite simplicial complex homeomorphic to the plane and satisfying a couple of other conditions is the nerve of a circle packing of either the plane or the disc (and not of both). We prove that such a complex is the nerve of a packing of the plane or the disc according as the simple random walk on its 1-skeleton is recurrent or transient, and discuss some applications. We also prove a criterion for transience of simple random walk on the 1-skeleton of a triangulation of the plane, in terms of average degrees of suitable sets of vertices.
\end{abstract}

\section{INTRODUCTION}

A circle packing is a collection $\mathcal{P}$ of circles, pairwise disjoint or tangent, such that the "gaps" between circles are "triangular"; more precisely, such that each component of the complement of the union of the closed discs corresponding to the circles of $\mathcal{P}$ has boundary made up of three circular arcs, one from each of three of the circles of $P$. We also require that the centres of the circles form a discrete set.

This definition makes sense in any space in which we know what is meant by "circle" and "closed disc", although when working in such generality one needs to make extra topological stipulations (for instance, the circles and the interstices between them must be simply-connected). For more details see [2]; these ideas aren't needed in this paper.

In this paper we shall be concerned with circle packings on the sphere, the plane and the hyperbolic plane. Notice that the notions of "circle" in these spaces agree. In fact we shall have little to say about the sphere; and we shall consider the hyperbolic plane as the unit disc in the plane, even to the extent of using the Euclidean metric.

To a circle packing $\mathcal{P}$ corresponds a certain 2-dimensional simplicial complex $K$, perhaps infinite (in what follows "complex" should be taken to mean "possiblyinfinite 2-dimensional simplicial complex"), as follows:

(1) There is one vertex of $K$ for each circle of $\mathcal{P}$.

(2) There is an edge joining two vertices iff their corresponding circles touch.

(3) There is a face (i.e., a 2 -simplex) meeting 3 vertices iff there is an "interstice" (i.e., a component of the complement of ..., as in the definition of circle packing) touching the corresponding circles.

Received by the editors August 19, 1994 and, in revised form, February 16, 1995.

1991 Mathematics Subject Classification. Primary 52C15; Secondary 30C35, 30G25, 60J15.

(C)1998 American Mathematical Society 
We call $K$ the nerve of $\mathcal{P}$. The nerve of $\mathcal{P}$ embeds in the underlying space of $\mathcal{P}$; put the vertices at the centres of the circles, and so on. In what follows we shall often, without further comment, identify $K$ with its image under this embedding.

It is a remarkable fact that the combinatorics of a packing, as "encoded" in its nerve, determine the geometry of the packing (its underlying space and how it is placed therein) to a very considerable degree. Beardon and Stephenson have proved in [2] the following theorem:

Theorem. A simply connected CP-complex without boundary is the nerve of a circle packing of exactly one of the three simply-connected Riemann surfaces.

(The precise definition of "CP-complex" is not relevant here. Roughly, a CPcomplex is a complex which might plausibly be the nerve of a reasonable circle packing. One fact which will be relevant in this paper is that, by definition, there is a uniform upper bound on degrees of vertices in a CP-complex. We say that $K$, or $\mathcal{P}$, has bounded degree.)

An obvious question now is: Which complexes correspond to packings of the plane, and which to packings of the disc? (A complex without boundary is the nerve of a packing of the sphere iff it is finite.) The main result of this paper is the following theorem.

Theorem 1. Let $R$ be either the plane or the disc, $K$ the nerve of a bounded-degree circle packing of $R$, and $X$ the 1-skeleton of $K$. Then $R$ is the plane iff the simple random walk on $X$ is recurrent, and the disc iff the simple random walk on $X$ is transient.

\section{SOME NECESSARY BACKGROUND}

We shall need the results in this section, but the trusting reader may simply read the statements of Facts $\mathrm{A}-\mathrm{C}$ and continue to the next.

Random walks. Let $X$ be a countable graph; suppose $X$ is connected (that is: the transitive closure of the adjacency relation relates every pair of vertices). To each ordered pair $\langle x, y\rangle$ of vertices let there be assigned a number $p_{x y}>0$ such that $\sum_{y} p_{x y}=1 \forall x$.

Let $x_{0}$ be any vertex of $X$, and consider the random variable $W$, whose values are infinite sequences of vertices of $X$, such that

(1) $W_{0}=x_{0}$;

(2) $\mathbb{P}\left(W_{n+1}=y \mid W_{n}=x\right)=p_{x y}$.

This is called the random walk on $X$ starting at $x_{0}$ with transition probabilities $\left(p_{x y}\right)$.

The matrix $P=\left(p_{x y}\right)$ of transition probabilities may be considered as operating on some suitable space of functions on $X$, thus: $\operatorname{Pf}(x)=\sum_{y} p_{x y} f(y)$. So $\operatorname{Pf}(x)$ is the expectation of $f\left(W_{1}^{x}\right)$ where $W^{x}$ is the random walk starting at $x$ with transition probabilities $p_{x y}$. (In the cases relevant to this paper, every vertex of $X$ is adjacent to only finitely many other vertices, so we can take the space to be that of finitelysupported functions from $X$ to $\mathbb{R}$; we shall do so.) If this operator is self-adjoint with respect to some positive measure $\lambda$ on $X$, we say that the random walk is reversible. What does this mean? First of all we need an inner product; for functions $f, g$ on $X$ 
write $\langle f, g\rangle:=\sum_{x} \lambda(x) f(x) g(x) . P$ is self-adjoint iff $\langle P f, g\rangle=\langle f, P g\rangle$ for all $f, g$. A little thought indicates that this happens iff $\lambda(x) p_{x y}=\lambda(y) p_{y x}$ for all $x, y$.

If $X$ is any graph, the simple random walk on $X$ is that with $p_{x y}=1 / \operatorname{degree}(x)$; that is, that in which we choose each possible move with equal probability at each stage. This is always reversible: take $\lambda_{x}=\operatorname{degree}(x)$.

The graphs in which we are interested have the additional property that there is a uniform upper bound on the degree of a vertex. This implies that the $\lambda(x)$ are uniformly bounded away from 0 and $\infty$, and that the $p_{x y}$ (for $x$ adjacent to $y$ ) are uniformly bounded away from 0 (of course they are also uniformly bounded away from $\infty$ !)

Diffusion. There is a parallel theory concerning things taking place on manifolds. Suppose we have a second-order strictly elliptic differential operator $L$ on a (connected) manifold $M$, and a positive measure $\sigma$ on $M$ w.r.t. which $L$ is self-adjoint. Then associated with $L$ is a "heat equation" $\partial \varphi / \partial t=L \varphi$, whose solutions may be thought of as describing diffusion on $M$. We may view this in a different way: associated to $L$ there is a semigroup of positive operators $T_{t}$ such that $T_{0}$ is the identity, and $f(x, t)=T_{t} \varphi(x)$ is a solution to $\partial f / \partial t=L f$. This gives us functions $p_{t}$ from $M \times M$ to $\mathbb{R}$ such that $T_{t}(x, d y)=p_{t}(x, y) d \sigma(y)$ (together with some other properties, such as the fact that $(y, t) \mapsto z=p_{t}(x, y)$ satisfies $\partial z / \partial t=L z$, this characterises the $p_{t}$ completely); from this it follows that $T_{t} f(x)=\int p_{t}(x, y) f(y) d \sigma(y)$ (compare the definition of the operator $P$ in the discrete case). These $p_{t}$ give rise to a Markov process on $M$, called diffusion on $M$.

The only cases that concern us in this paper have $M$ a subset of $\mathbb{R}^{2}$ and $L=-\Delta$, with $\Delta$ being the Laplacian. In this case diffusion is just Brownian motion.

Recurrence and transience. In either of these scenarios there is a dichotomy between "recurrence" and "transience". We may define the Green's function by $G(x, y)=\int_{0}^{\infty} p_{t}(x, y) d t$ in the continuous case and $G(x, y)=\sum p_{x y}^{(n)}$ in the discrete case (where $p_{x y}^{(n)}$ is the " $n$-step transition probability", with the obvious definition); then either $G(x, y)$ is always finite (for $x \neq y$ ) or it is always infinite; in the former case we say that $X$ (or $M$ ) is transient, in the latter that it is recurrent. (Of course this should refer to $\langle X, P, \lambda\rangle$ or to the corresponding thing in the continuous case; the abuse of notation here exemplified will be repeated.)

Alternatively, we say that $X$ is transient iff there is a non-zero probability that the random walk starting at $x_{0}$ never returns to $x_{0}$. (This does not depend on our choice of $x_{0}$.) And we say that $M$ is transient iff there is a non-zero probability that either (1) the "lifetime" of the diffusion process on $M$ starting at any point $x_{0}$ is finite, or (2) there is some non-empty open set such that the times at which it is visited are bounded. Again, this doesn't depend on $x_{0}$.

Alternatively we can think more directly in terms of solutions of the heat equation. Let $\varphi$ be a (reasonably nice) function on $M$ such that $\int \varphi d \sigma=1$ and $\varphi>0$. There is a unique solution $F$ of the heat equation with $F(x, 0)=\varphi(x)$; and $M$ is transient iff $\forall x \int_{0}^{\infty} F(x, t) d t<\infty$. (It doesn't much matter exactly what $\varphi$ we choose. Noting that $F(x, t)=T_{t} \varphi(x)$, the relation with our first definition is apparent.)

These definitions are all equivalent, and we need not say which we shall use because everything will be done in terms of the conditions given in the next section.

An easy and important Fact: the disc is transient, and the plane is recurrent. 
The Dirichlet criterion. Let $S$ be either $X$ or $M$, and suppose we have one of the two situations just described. (So we have an operator $1-P$ or $L$, and an invariant measure $\lambda$ or $\sigma$.) We may define a Dirichlet form, a symmetric bilinear form on a suitable subspace of $L^{2}(S)$, thus:

(1) $\mathcal{E}(\varphi, \psi):=\int \varphi L \psi d \sigma$ in the continuous case;

(2) $\mathcal{E}(f, g):=\sum f(x)[(1-P) g](x) \lambda(x)$ in the discrete case.

It turns out that the following propositions are true, where $C_{c}(X)$ is the space of finitely-supported real functions on $X$, and $C_{c}^{2}(M)$ is the space of compactlysupported twice continuously differentiable functions on $M$ :

Proposition. $(X, P)$ is transient iff there exist $x_{0} \in X$ and a constant $C$ such that

$$
\forall f \in C_{c}(X) \quad f\left(x_{0}\right)^{2} \leqslant C \cdot \mathcal{E}(f, f),
$$

iff there is a positive function $\alpha$ on $X$ such that

$$
\forall f \in C_{c}(X) \quad \sum \alpha f^{2} \leqslant \mathcal{E}(f, f) .
$$

Proposition. $(M, L)$ is transient iff there exist a non-empty open set $U \subseteq M$ and a constant $C$ such that

$$
\forall \varphi \in C_{c}^{2}(M) \quad\left(\int_{U} \varphi\right)^{2} \leqslant C \cdot \mathcal{E}(\varphi, \varphi)
$$

iff there is a positive measurable function $\beta$ on $M$ such that

$$
\forall \varphi \in C_{c}^{2}(M) \quad \int_{M} \beta \varphi^{2} \leqslant \mathcal{E}(\varphi, \varphi) .
$$

Proposition. Given any $(X, P)$ as above, and any $f \in C_{c}(X), \mathcal{E}(f, f)$ is within a constant factor of $\sum(f(x)-f(y))^{2}$, the sum being taken over all pairs of adjacent vertices $x, y$.

Proposition. For $\varphi \in C_{c}^{2}(M), \quad-\int_{M} \varphi \Delta \varphi d \sigma=\int_{M}\|\nabla \varphi\|^{2}$.

The first two of these may be found in [1]; the fourth (in the statement of which we have already assumed that $M$ is an open subset of some Euclidean space) is a simple application of the divergence theorem. The third is stated in [1]; it is true because (by the remarks at the end of section 3.1 there) $\mathcal{E}(f, f)$ is within a constant factor of $\sum f(x)(f(x)-f(y))$, which equals (by change of variables) $\sum f(y)(f(y)-f(x))$; and the sum of these is exactly the expression we require.

Incidentally, there is an unfortunate lack of uniformity among the world's mathematicians regarding the meaning of "positive". In the statements above, and in the rest of this paper, it means " $>0$ " rather than " $\geqslant 0$ ".

In other words... We are really only interested in a very special case of all this. The four Propositions we have just stated yield the following two facts, on which our proof of Theorem 1 will be based. " $R$ " here, of course, takes the place of " $M$ " above.

Fact $\mathbf{A}$. The simple random walk on $X$ is transient iff there exist a vertex $x_{0}$ of $X$ and a constant $C$ such that, for all $f \in C_{c}(X)$,

$$
f\left(x_{0}\right)^{2} \leqslant C \cdot \sum(f(x)-f(y))^{2}
$$


where the RH summation is over all pairs of adjacent vertices $x, y$; this is in turn equivalent to the existence of a positive function $\alpha$ on $X$ such that, for all $f$,

$$
\sum \alpha f^{2} \leqslant \sum(f(x)-f(y))^{2} .
$$

Fact B. $R$ is transient iff there exist a non-empty open $U \subseteq R$ and a constant $C$ such that, for all $\varphi \in C_{c}^{2}(R)$,

$$
\left(\int_{U} \varphi\right)^{2} \leqslant C \cdot \int_{R}\|\nabla \varphi\|^{2}
$$

this is in turn equivalent to the existence of a positive measurable function $\beta$ on $M$ such that, for all $\varphi$,

$$
\int_{R} \beta \varphi^{2} \leqslant \int_{R}\|\nabla \varphi\|^{2}
$$

The Ring Lemma. The following result, which is of considerable importance, is proved in [5].

Theorem ("Ring Lemma"). If a circle in a circle packing has radius $r$ and $n$ neighbours, all its neighbours have radius at least $c(n) r$, where $c(n)$ is a positive constant depending only on $n$.

An immediate consequence of this is

Fact C. In a circle packing of bounded degree there is a uniform bound on the ratio of the radii of two adjacent circles.

\section{Proof of Theorem 1}

We shall prove first that if $R$ is the disc, then $X$ is transient. In the next section we shall prove the converse implication.

If $R$ is the disc, then (2b) holds; we shall show that (1b) holds too. Consider any function $f$ as in (1b), and define a function $\varphi$ by making $\varphi(x)=f(x)$ when $x$ is a vertex of $K$ and interpolating linearly on the faces of $K$. (The union of the faces of $K$ is all of $R$.) Actually the result of doing this is not $C^{2}$, but we can make it $C^{2}$ while making arbitrarily small changes to the integrals in (2b); so we'll pretend it is.

Now consider any face of $K$, with vertices at $x, y, z$. Scale, rotate and translate it so that the vertices of the new triangle are at $x_{1}=(0,0), y_{1}=(1,0)$ and $z_{1}=(p, q)$; Fact C implies that $p, q$ are bounded away from $0, \infty$. Denote by $\varphi_{1}$ the linear function on this new triangle with $\varphi_{1}\left(x_{1}\right)=\varphi(x)=f(x), \varphi_{1}\left(y_{1}\right)=\varphi(y)=f(y)$ and $\varphi_{1}\left(z_{1}\right)=\varphi(z)=f(z)$. Write $A:=(f(y)-f(z))^{2}$ and similarly for $B, C$. Then an easy calculation shows that on this transformed triangle (call it $T$ )

$$
\left\|\nabla \varphi_{1}\right\|^{2}=\frac{p}{q^{2}} A+\frac{1-p}{q^{2}} B+\left(1-\frac{p(1-p)}{q^{2}}\right) C,
$$

whence (since the area of $T$ is $q / 2$ )

$$
\int_{T}\left\|\nabla \varphi_{1}\right\|^{2}=\frac{p}{2 q} A+\frac{1-p}{2 q} B+\left(\frac{q}{2}-\frac{p(1-p)}{2 q}\right) C .
$$

What is the integral of $\left\|\varphi^{2}\right\|$ over the original triangle? Suppose the scaling factor to have been $\lambda$; that is, suppose that the new triangle is $\lambda$ times the (linear) 
size of the old one. Then $\left\|\nabla \varphi_{1}\right\|=\|\nabla \varphi\| / \lambda$, but the area of the new triangle is $\lambda^{2}$ times that of the old one; so the two integrals are the same.

Now, the RHS here is at most some constant times $(f(x)-f(y))^{2}+(f(y)-f(z))^{2}$ $+(f(z)-f(x))^{2}$; for $q$ is bounded away from 0 and $\infty$, and $p$ is bounded away from $\infty$. (Incidentally, it also happens to be bounded below by some constant times the same thing, though this requires proof.)

Now sum over all faces of $K$. Each $(f-f)^{2}$ term occurs precisely twice; and all of $R$ is covered exactly once (apart from a set of measure zero, along the edges of the triangles). Hence

$$
\int_{R}\|\nabla \varphi\|^{2} \leqslant \mathrm{cst} \cdot \sum(f(x)-f(y))^{2}
$$

Here the constant depends only on our bounds on $p, q$ above, and hence ultimately only on the bound for degrees of vertices in the packing (via the Ring Lemma).

We must also consider the LH sides of (1b) and (2b). The result we need here is the following:

Lemma. Let $\beta$ be a positive function on $R$, and write $r_{x}$ for the radius of the circle at $x$. Let $\beta_{1}(x)=\inf \beta$ where the infimum is taken over the union of the faces of $K$ meeting $x$ (note that by compactness the infimum is attained and hence non-zero). Then

$$
\sum \beta_{1}(x) r_{x}^{2} f(x)^{2} \leqslant \operatorname{cst} \cdot \int_{R} \beta \varphi^{2}
$$

Proof. Consider first a single face $T$. I claim that

$$
\int_{T} \varphi^{2} / \operatorname{area}(T)=\frac{1}{12}\left\{f(x)^{2}+f(y)^{2}+f(z)^{2}+(f(x)+f(y)+f(z))^{2}\right\} \geqslant \frac{1}{12} f(x)^{2} .
$$

The inequality is trivial; for the equality, note that the LHS is not affected by any affine transformation (think about the formula for change of variable and its relation to area), whence we need only prove it for one particular triangle. Consider a triangle with vertices at $(0,0),(1,0)$ and $(0,1)$; now the expression for $\varphi$ can be written down immediately and the integration is an easy one.

But now we have

$$
\begin{aligned}
\int_{R} \beta \varphi^{2}=\sum_{T} \int_{T} \beta \varphi^{2}=\frac{1}{3} \sum_{(x, T)} \int_{T} \beta \varphi^{2} & \geqslant \frac{1}{3} \sum_{(x, T)} \beta_{1}(x) \int_{T} \varphi^{2} \\
& \geqslant \frac{1}{3} \sum_{(x, T)} \beta_{1}(x) \frac{1}{12} f(x)^{2} \cdot \operatorname{area}(T) \\
& \geqslant \frac{1}{36} \sum_{x} \beta_{1}(x) K r_{x}^{2} \cdot f(x)^{2}
\end{aligned}
$$

where the summations over $(x, T)$ are over all pairs for which $x$ is a vertex of $T$, and the lemma is proved. (The last inequality holds because of Fact C.)

So

$$
\sum \beta_{1}(x) r_{x}^{2} f(x)^{2} \leqslant \mathrm{cst} \cdot \int \beta \varphi^{2} \leqslant \mathrm{cst} \cdot \int\|\nabla \varphi\|^{2} \leqslant \mathrm{cst} \cdot \sum(f(x)-f(y))^{2} ;
$$

in other words, writing $\alpha(x)$ for a suitable constant times $\beta_{1}(x) r_{x}^{2}$, the condition (1b) holds; so the simple random walk on $X$ is transient. 
Second half of proof. For the other half of the theorem, let us suppose that the random walk is transient, so that (1a) holds (for some choice of $x_{0}$ and $C$ ) for all $f \in C_{c}(X)$.

Let $\varphi$ be any $C_{c}^{2}$ function on $R$; we shall prove that (2a) holds, where $U$ is the union of the faces of $K$ meeting $x_{0}$ and the constant is chosen appropriately. Define a function $f$ on $X$ as follows: $f(x)$ is the average of $\varphi$ over all the faces of $K$ meeting $x$. (In particular, $f\left(x_{0}\right)$ is precisely $\int_{U} \varphi / \operatorname{area}(U)$.)

By the parenthetical remark just made, we shall be done if we can show that the RHS of (1a) is bounded above by some constant multiple of the RHS of (2a).

Let $x$ and $y$ be two adjacent vertices; write $A$ and $B$ for the sets over which $\varphi$ is averaged to obtain $f(x)$ and $f(y)$. Then

$$
f(x)-f(y)=\int_{A} \int_{B} \varphi(a)-\varphi(b) d a d b /|A||B|
$$

(if this is not obvious, express the RH side as the difference of two repeated integrals). Let $D$ be the smallest disc containing both $A$ and $B$; the absolute value of the $\mathrm{RH}$ side of this last equation is at most

$$
\begin{aligned}
\frac{1}{|A||B|} \iint_{D \times D}|\varphi(a)-\varphi(b)| d a d b & \leqslant \frac{1}{|A||B|} \iint_{D \times D} \int_{a}^{b}\|\nabla \varphi(v)\| d v d a d b \\
& =\frac{1}{|A||B|} \int_{D}\|\nabla \varphi(v)\|\left|E_{v}\right| d v \\
& =\frac{1}{|A||B|} \int_{D}\|\nabla \varphi(v)\| \int_{D}\left|E_{v, a}\right| d a d v \\
& \leqslant \frac{1}{|A||B|} \cdot|D| \operatorname{diameter}(D) \int_{D}\|\nabla \varphi(v)\| d v
\end{aligned}
$$

where $E_{v}=\{(a, b) \in D \times D: v \in[a, b]\}$ and $E_{v, a}=\{b: v \in[a, b]\}$.

Noting that $|A|,|B|$ and $|D|$ are all within constant factors of each other by Fact $\mathrm{C}$, and further that $|D|$ is a constant times diameter $(D)^{2}$, this yields

$$
(f(x)-f(y))^{2} \leqslant \operatorname{cst} \cdot \frac{1}{|D|}\left(\int_{D}\|\nabla \varphi(v)\|\right)^{2} \leqslant \operatorname{cst} \cdot \int_{D}\|\nabla \varphi\|^{2} .
$$

Sum this over all pairs of adjacent vertices. The $\mathrm{RH}$ side of the result is within a constant factor of $\int_{R}\|\nabla \varphi\|^{2}$ by Fact C. And now condition (2a) holds, as required: for

$$
\left(\int_{U} \varphi\right)^{2} \leqslant \operatorname{cst} \cdot f\left(x_{0}\right)^{2} \leqslant \operatorname{cst} \cdot \sum(f(x)-f(y))^{2} \leqslant \operatorname{cst} \cdot \int\|\nabla \varphi\|^{2}
$$

so $R$ must be the disc. This concludes the proof.

\section{Applications}

Theorem 1 is nice to have, but not especially useful in practice; after all, it's not all that easy to tell whether a given network is transient or recurrent. However, there are some theorems relating growth conditions of one sort or another to transience/recurrence, and these can be useful. As an example of the sort of thing that can be done, we prove the following. 
Theorem 2. Let $r>1$ and $\delta>0$, and let $K$ be a $C P$-complex such that for any union $A$ of combinatorial balls of radius $r$ the average degree of all vertices in $A$ is at least $6+\delta$. Then the simple random walk on its 1-skeleton is transient; hence the associated circle packing fills the disc rather than the plane.

Proof of Theorem 2. We shall need some definitions. For $A$ a set of vertices of $K$, the boundary $\partial A$ of $A$ is the set of vertices in $A$ with neighbours in $K-A$. The total discrepancy $D(A)$ is $\sum_{x \in A}($ degree $(x)-6)$.

Write $B_{r}(x)$ for the combinatorial ball of radius $r$ about $x$; that is, $B_{r}(x):=$ $\{y: d(x, y) \leqslant r\}$. Our condition, then, implies that $D\left(B_{r}(x)\right) \geqslant \delta\left|B_{r}(x)\right|$ for all $x$. I shall write hereafter " $r$-ball" for "combinatorial ball of radius $r$ ".

We shall also need the constant $N_{r}$, defined to be an upper bound on the number of vertices in any $r$-ball. If $d$ is the maximum degree of any vertex, we may take $N_{r}=d^{r}$.

The proof now consists of a chain of easy lemmas; the idea is to establish an isoperimetric inequality for arbitrary sets of vertices in $K$.

Let $\hat{A}$ be the subcomplex of $K$ spanned by $A$; that is, the subcomplex of $K$ consisting of the vertices in $A$, the edges between them and the faces spanned by them.

Lemma. Suppose $\hat{A}$ is a connected subcomplex of $K$. Then

$$
|\partial A| \geqslant D(A) /(6+d) \text {. }
$$

Proof. Let $f, e, v$ be the number of faces, edges, vertices of $\hat{A}$. Write $\operatorname{deg}_{0}(x)$ for the degree in $\hat{A}$ of vertex $x$; then $2 e=\sum_{A} \operatorname{deg}_{0}$. Also (by a counting argument) $3 f=2 e-n_{\partial}-n_{\partial \partial}$, where $n_{\partial}$ is the number of boundary edges of $\hat{A}$ and $n_{\partial \partial}$ is the number of edges of $\hat{A}$ not in any face of $\hat{A}$. Also, the Euler characteristic of $\hat{A}$ is just $2-k$ where $k$ is the number of components of $K-\hat{A}$. Since plainly $n_{\partial}, n_{\partial \partial} \geqslant 0$ and $k \leqslant|\partial \hat{A}|$, we have by Euler's formula

$$
\sum_{A}\left(\operatorname{deg}_{0}-6\right)=-6 \chi-2 n_{\partial}-2 n_{\partial \partial} \leqslant-12+6|\partial A| .
$$

But now note that the LHS here is $D(A)-\sum_{A} d_{\text {out }}$ where $d_{\text {out }}(x)$ is the number of edges meeting $x$ which do not lie in $\hat{A}$-so $\sum_{A} d_{\text {out }} \leqslant d|\partial A|$. Putting all this together we obtain

$$
\begin{aligned}
d|\partial A| & \geqslant \sum_{A} d_{\text {out }}=D(A)-\sum_{A}\left(\operatorname{deg}_{0}-6\right) \\
& \geqslant D(A)+12-6|\partial A| \geqslant D(A)-6|\partial A|,
\end{aligned}
$$

or in other words $|\partial A| \geqslant D(A) /(6+d)$ as required.

Lemma. Suppose every union of $r$-balls has average degree at least $6+\delta$. Then every union $A$ of $r$-balls has $|\partial A| \geqslant \delta_{1}|A|$.

Proof. Each boundary point is a boundary point of at most $d$ components of $\hat{A}$, so it is enough to do this under the assumption that $\hat{A}$ is connected. But this is just the previous lemma.

Lemma. Suppose every union of $r$-balls has $|\partial A| \geqslant \delta_{1}|A|$. Then every set has $|\partial A| \geqslant \delta_{2}|A|$. 
Proof. Let $A$ be any set of vertices. If the number of vertices in $A$ which are not in any $r$-ball contained in $A$ is at least $\varepsilon|A|$ (note: we shall choose $\varepsilon$ in a moment), then $A$ already satisfies an inequality of the desired type, for there is a boundary point within $r$ of any such vertex, and the number of vertices corresponding to each boundary point is at most $N_{r}$. So we may assume that the number of such vertices is less than $\varepsilon|A|$. Let $A_{1}$ be the set obtained from $A$ by adding the $r$-ball about each such vertex; the resulting set $i s$ a union of $r$-balls, so $\left|\partial A_{1}\right| \geqslant \delta_{1}\left|A_{1}\right|$; and $\left|\partial A_{1}\right|-|\partial A| \leqslant N_{r} \varepsilon|A|$; so, taking $\varepsilon=\delta_{1} / 2 N_{r}$ the result follows.

Finally we can put all this together to deduce

Theorem. Let $K$ be a CP-complex such that the average degree within each union of $r$-balls is at least $6+\delta$. Then it satisfies an isoperimetric inequality of the form " $\partial A|\geqslant c| A \mid$ for all sets of vertices $A$ ".

From this Theorem 2 is an immediate corollary on applying Theorem 2.4 of [3], which says precisely that a graph of bounded degree satisfying an isoperimetric inequality is transient.

Incidentally, Dodziuk proves in [3] that any graph all of whose degrees are $\geqslant 7$ satisfies an isoperimetric inequality (and so is transient). His method of proof is broadly similar to mine above.

As it happens, any triangulation of the plane with bounded degree is a CPcomplex. So we can restate Theorem 2 thus:

Theorem 3. A triangulation of the plane with a uniform bound on degrees is transient provided there are $r>1$ and $\delta>0$ such that the average degree of vertices within any union of $r$-balls is at least $6+\delta$.

(This appears to be new; certainly I have not seen it elsewhere, though it wouldn't surprise me to find it has been proved already.)

Some other "average-degree" results. It's actually possible to improve Theorem 3 considerably, using a result of Thomassen [6]. Define the discrepancy of a subset of our triangulation to be $\sum$ degree -6 ; then we have

Theorem 4. A triangulation of the plane with a uniform bound on degrees is transient provided that, for some $r$, the discrepancy of any union $A$ of combinatorial balls of radius $r$ is at most $f(|A|)$ where $f$ is an increasing positive function with $\sum f^{-2}<\infty$.

In the other direction,

Theorem 5. A triangulation of the plane with a uniform bound on degrees is recurrent provided that, for some vertex $x_{0}$ and all $n$, the discrepancy of the combinatorial ball about $x_{0}$ of radius $n$ is at least $f(n)$, where $f$ is an increasing positive function with $\sum f^{-1}=\infty$.

And in the other other direction,

Theorem 6. For any increasing positive function $f$ with $f(n+1) / f(n)<\sqrt{2}$ and $\sum f^{-1}<\infty$, there is a counterexample to the corresponding version of Theorem 5 .

At present I can't give any reference for these other than [4]. 
A few unanswered questions. All the proofs in this paper make use of the assumption that our complexes have bounded degree. The main theorem seems to require this very strongly; is it actually true without the assumption? (My guess: no.) What about Theorem 3? (My guess: yes.)

Theorem 2 is a bit unsatisfactory: does it remain true if "any union of combinatorial balls of radius $r$ " is replaced by "any combinatorial ball of radius at least $r$ "? (My guess: yes.)

Note. Oded Schramm and Zheng-Xu He have recently proved that Theorem 1 holds in greater generality. In particular, they have answered the first unanswered question above: the answer is "not quite, but almost". (Their work is entirely independent of mine.)

\section{REFERENCES}

[1] A. Ancona, Théorie du potentiel sur les graphes et les varietés, Ecole d'Eté de Probabilités de Saint-Flour XVIII, 1988, pp. 5-116.

[2] A. F. Beardon \& K. Stephenson, The uniformization theorem for circle packings, Indiana U. Math. J. 39 (1990), 1383-1425. MR 92b:52038

[3] J. Dodziuk, Difference equations, isoperimetric inequality and transience of certain random walks, Trans. American Math. Soc. 284 (1984), 787-794. MR 85m:58185

[4] G. J. McCaughan, Some results on circle packings, Ph.D. thesis, University of Cambridge, 1996.

[5] B. Rodin \& D. Sullivan, The convergence of circle packings to the Riemann mapping, J. Differential Geom. 26 (1987), 349-360. MR 90c:30007

[6] C. Thomassen, Isoperimetric inequalities and transient random walks on graphs, Ann. Prob. 20 (1992), 1592-1600. MR 94a:60106

Department of Pure Mathematics and Mathematical Statistics, Cambridge UniverSity, Mill Lane, Cambridge, England

E-mail address: gjm11@pmms.cam.ac.uk 